\title{
Growth of GaN on Si(111): Surfaces and crystallinity of the epifilms and the transport behavior of GaN/Si heterojunctions
}

\author{
Zhongjie Xu, ${ }^{1}$ Lixia Zhang, ${ }^{2}$ Hongtao He, ${ }^{2}$ Jiannong Wang, ${ }^{2}$ and Maohai Xie ${ }^{1, a)}$ \\ ${ }^{1}$ Physics Department, The University of Hong Kong, Pokfulam Road, Hong Kong \\ ${ }^{2}$ Physics Department, Hong Kong University of Science and Technology, Clear Water Bay, \\ Kowloon, Hong Kong
}

(Received 24 May 2011; accepted 6 October 2011; published online 9 November 2011)

\begin{abstract}
Growths of $\mathrm{GaN}$ on $\mathrm{Si}(111)-(7 \times 7)$ substrates by plasma-assisted molecular-beam epitaxy (PA-MBE) have been studied. Optimal conditions of MBE and the effect of a low-temperature (LT) buffer are followed. It is found that irrespective of the growth conditions and the growth strategies (direct versus two-step growth), a thin amorphous-like interface layer always forms. For smooth surfaces and better crystallinity of the epifilms, a LT-buffer preceding the high-temperature deposition is helpful, and the grown GaN films are of nitrogen-polar. Transport measurements of the heterojunctions of GaN on heavily $p$ - and $n$-doped Si reveal ohmic behavior, whereas that of $n$-GaN on lightly doped $n^{-}$-Si substrate shows rectifying characteristics. (C) 2011 American Institute of Physics. [doi:10.1063/1.3658850]
\end{abstract}

\section{INTRODUCTION}

III-nitrides are proven semiconductors for modern optoelectronic and microelectronic applications, including light-emitting devices (LEDs) and field-effect transistors (FETs). ${ }^{1-4}$ Their potentials for solar cell applications have received increasing attention in recent years, as the bandgaps of $(\mathrm{Al}, \mathrm{Ga}, \mathrm{In}) \mathrm{N}$ match almost perfectly with the solar spectrum. $^{5-7}$ Indeed, a high power conversion efficiency is expected for multijunction tandem cells made of InGaN. ${ }^{7-11}$ Theoretically, a maximum power conversion efficiency of over $70 \%$ was predicted for a nitride-based tandem cell. ${ }^{12}$ Practically, however, making a fully nitride-based high efficiency tandem cell is difficult. This is partly related to the relatively inferior quality of nitride crystals grown epitaxially on some heterogeneous substrates, such as $\mathrm{SiC}$ and sapphire, as well as to the problems associated with $p$-type doping in nitrides.

To circumvent the problems associated with $p$-type doping in nitrides, it has been proposed to grow readily attainable $n$-type $\operatorname{In}_{x} \mathrm{Ga}_{1-x} \mathrm{~N}$ epilayers on $p$-type $\mathrm{Si}$ or Ge substrate, so that heterogeneous $p-n$ junctions are created for photovoltaic characteristics. ${ }^{5,13,14} \mathrm{Si}$ and $\mathrm{Ge}$ are attractive because of the low cost as well as the well-established processing technology. In 2003, Yamaguchi et al. experimented depositing $\mathrm{InN}$ and $\mathrm{InGaN}$ on $p$-type $\mathrm{Si}(111)$ by plasmaassisted molecular-beam epitaxy (PA-MBE) and observed the rectifying behavior of the junctions, ${ }^{5,13-15}$ whereas Trybus et al. studied $n$-InN/p-Ge junction but observed no rectifying property, probably due to charge accumulation at the interface layers. ${ }^{16,17}$

Different from epitaxial nitride layers for FETs and LEDs, where only the top region of the epifilms are deviceactive, photovoltaic devices operate at the very interface

${ }^{\text {a)} E l e c t r o n i c ~ m a i l: ~ m h x i e @ h k u . h k . ~}$ region of the heterojunction. Therefore, strategies used earlier for obtaining high quality epitaxial nitride films on $\mathrm{Si}$, such as epitaxial lateral overgrowth ${ }^{18,19}$ and Pendeoepitaxy, ${ }^{20}$ may not be applicable for growing solar cell structures. Yet, due to a chemical dissimilarity between IIInitrides and $\mathrm{Si}$, as well as the large lattice and thermal mismatches between the two, deposition of nitrides on clean $\mathrm{Si}$ surface can be inherently difficult. Strain relaxation inevitably leads to defect formation, degrading the transport properties of the junction. Therefore, it is challenging to achieve high quality $\mathrm{GaN} / \mathrm{Si}$ junctions for high performance solar cells.

For direct growth of III-nitrides on unpatterned $\mathrm{Si}$, a thin crystalline $\mathrm{Si}_{3} \mathrm{~N}_{4}$ layer formed by a nitridation process of the substrate has been shown beneficial for subsequent deposition of GaN. ${ }^{21-23}$ Alternatively, one may resort to a metallic surfactant layer or some novel buffer techniques. ${ }^{24-27}$ Although these techniques are said to lead to improved epitaxial films, their superiority in photovoltaic applications remains to be demonstrated.

In this study, we carry out a systematic investigation of GaN growth on clean $\mathrm{Si}(111)-(7 \times 7)$ without a priori surface treatment. We find the two-step growth procedure is helpful in obtaining single crystalline epifilms with smooth surface morphologies. Based on reflection high-energy electron diffraction (RHEED) observations of the growing fronts, we establish the optimal thickness of the low-temperature (LT) buffer layer in the two-step growth procedure. The grown GaN films are all of nitrogen (N)-polar, i.e., the topmost layer of atoms of the grown $\mathrm{GaN}$ are nitrogen. Examinations by transmission electron microscopy (TEM) reveal the presence of an amorphous-like layer at GaN/Si interface, which is not caused by the LT-growth procedure. Growth without employing a LT-buffer even results in a thicker amorphous-like layer at the heterointerface. Transport properties of $\mathrm{GaN} / \mathrm{Si}$ junctions are characterized, which reveal the rectifying behavior only for $\mathrm{GaN} / n^{-}-\mathrm{Si}$ junctions. 


\section{EXPERIMENT}

The growth experiments were conducted in a PA-MBE reactor equipped with Knudsen cells for elemental gallium (Ga) and a radio-frequency plasma unit (OAR HD-25) for nitrogen flux. The latter was operated at powers of 100-300 Ws under a $\mathrm{N}_{2}$ flow rate of $\sim 0.2 \mathrm{sccm}$ (standard cubic centimeters per minute), delivering fluxes of active $\mathrm{N}$ spices (mostly neutral $\mathrm{N}$ atoms according to plasma spectrum analysis) in the range of $1.1-3.4 \times 10^{14} \mathrm{~cm}^{-2} \mathrm{~s}^{-1}$, or equivalently $0.05-0.15 \mathrm{GaN}$ bilayers per second (BLs/s, where 1 $\mathrm{BL}=c / 2 \approx 2.6 \AA$ with $c$ being the lattice constant of $\mathrm{GaN}$ along [0001], the growth direction of the crystal). The flux of Ga was controlled by the cell temperature, which was varied for achieving different $\mathrm{Ga}: \mathrm{N}$ flux ratios. In this investigation, the deposition rate of the film was maintained the same, which was $\sim 0.05 \mathrm{BLs} / \mathrm{s}$, as determined by either $\mathrm{N}$ or $\mathrm{Ga}$ flux in the Ga- or $\mathrm{N}$-stable regimes, respectively. ${ }^{28}$ The growing surfaces were monitored in real time by the RHEED operating at $10 \mathrm{keV}$, where the diffraction patterns were captured by a charge-coupled device camera or a commercial digital camera mounted on the phosphors screen. Both $p$ and $n$-type $\mathrm{Si}(111)$ substrates were adopted for GaN epitaxy. The commercial Si wafers were cut into $4 \times 11 \mathrm{~mm}^{2}$ rectangular pieces followed by ultrasonic degreasing and cleaning in solvents and deionized water repeatedly before being loaded into the ultrahigh vacuum (UHV) system for degassing at $\sim 500{ }^{\circ} \mathrm{C}$ for several hours. Deoxidization of the substrate was achieved by thermal flashing to temperatures as high as $\sim 1200{ }^{\circ} \mathrm{C}$ through a direct current flowing through the long side of the rectangular sample, after which clean and $(7 \times 7)$ reconstructed $\mathrm{Si}(111)$ surface was obtained as characterized by the RHEED [Fig. 1(i)]. Afterward, the sample temperature was brought down to some specified values $\left(400-800^{\circ} \mathrm{C}\right)$ for $\mathrm{GaN}$ deposition. To initiate $\mathrm{GaN}$ growth, shutters of $\mathrm{Ga}$ and $\mathrm{N}$ cells were opened simultaneously immediately after the N-plasma was ignited. A short time inter-

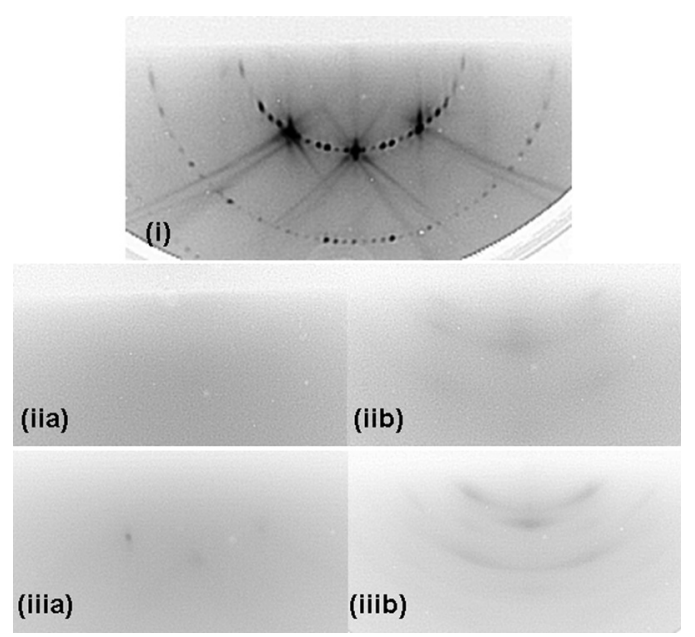

FIG. 1. RHEED patterns taken along GaN [1120] direction from the surfaces of epitaxial GaN-on-Si(111) at nominal thicknesses of (a) 5 BLs and (b) 20 BLs. Panels (ii) and (iii) refer to growth at temperatures of 400 and $650{ }^{\circ} \mathrm{C}$, respectively, while the RHEED pattern (i) is from $\mathrm{Si}(111)-(7 \times 7)$ substrate surface. val between N-plasma ignition and shutter-opening was essential to prevent unintentional nitridation of $\mathrm{Si}$. In this experiment, we ensured the $(7 \times 7)$ pattern of the substrate to be observable by the RHEED at the time of source shutter opening. For comparison, in some growth runs, a pretreatment of $\mathrm{Si}(111)-(7 \times 7)$ surface by different Ga dosage was conducted so as to minimize Si nitridation. However, little change was found comparing to those grown on clean $\mathrm{Si}(111)$. For the two-step growth procedure, an initial LT $\left(400^{\circ} \mathrm{C}\right)$ buffer layer was deposited for a specified thickness, followed by a $\mathrm{HT}\left(650^{\circ} \mathrm{C}\right)$ deposition of a thicker film on top.

The evolution of the surface as well as the crystallinity of the epifilm at different growth stages were monitored and evaluated by the RHEED. Particularly the width of the diffraction streaks/spots and the interspot/streak spacing were recorded. The grown samples were also characterized ex situ by scanning electron microscopy (SEM), transmission electron microscope (TEM) and x-ray diffraction (XRD) for morphological and structural information. For the purpose of transport study, the concentrations of electrical carries in the substrates ( $p$ - and $n$-Si) and in epifilms were characterized by the Hall-effect measurements. Ohmic contacts to $n$-GaN were made by depositing Al or ITO (indium tin oxide), while those to $p$ - and $n$-Si were achieved by depositing $\mathrm{Al}$ and $\mathrm{Ag}$, respectively. For the Hall measurement of epitaxial GaN films, van der Pauw geometry with the electrodes deposited on top of the films grown on lightly $n$-doped substrates are adopted so as to minimize errors introduced by parallel conduction through the substrate. Indeed, the measured electron doping in such GaN films ranged from $4 \times 10^{18} \mathrm{~cm}^{-3}$ to $1.4 \times 10^{20} \mathrm{~cm}^{-3}$ (depending on the conditions of MBE), order of magnitude higher than that in the lightly $n$-doped $\mathrm{Si}$ substrate $\left(\sim 2.0 \times 10^{15} \mathrm{~cm}^{-3}\right)$. The current-voltage $(I-V)$ characteristics of $\mathrm{GaN} / \mathrm{Si}$ heterojunctions were measured at temperatures in the range of $18-400 \mathrm{~K}$.

\section{RESULTS AND DISCUSSIONS}

The MBE conditions, such as the growth temperature and $\mathrm{Ga} / \mathrm{N}$ flux ratio, are first optimized for better surfaces and crystallinity of epitaxial GaN films grown directly on $\mathrm{Si}(111)-(7 \times 7)$. Afterwards, a two-step growth procedure is explored, where the effect of the LT-buffer thickness is followed by observations of the surfaces during the early state depositions.

\section{A. Effect of temperature and flux}

To achieve better surfaces of crystalline GaN grown directly on $\mathrm{Si}(111)-(7 \times 7)$, we first carried out an optimization of the growth conditions of MBE. Figure 1 presents a series of the RHEED patterns taken at epifilms' thicknesses of (a) $5 \mathrm{BLs}$ and (b) $20 \mathrm{BLs}$, but grown at (ii) $400^{\circ} \mathrm{C}$ and (iii) $650^{\circ} \mathrm{C}$, respectively. The RHEED pattern of the starting $\mathrm{Si}(111)-(7 \times 7)$ surface is given in (i). In this set of samples, the flux ratio between $\mathrm{Ga}$ and $\mathrm{N}$ has been kept at $0.88: 1$, so the growth was in the so-called N-stable regime. ${ }^{28}$ Growth at higher temperatures, e.g., $800^{\circ} \mathrm{C}$, were also 
attempted, but the RHEED shows no GaN nucleation but a dominant nitridation process of $\mathrm{Si}$ as characterized by the appearance of a $(8 \times 8)$ pattern. ${ }^{29}$ At temperatures below $650^{\circ} \mathrm{C}$, we observe the surfaces quickly become amorphouslike upon the initiation of $\mathrm{GaN}$ deposition as characterized by diffusive RHEED patterns shown in Fig. 1(iia) and (iiia). However, at $650^{\circ} \mathrm{C}$, a ring pattern emerges at a deposition thickness of $\sim 20$ BLs [Fig. 1(iiib)], suggesting a transition from an amorphous layer to a polycrystalline film as the deposition proceeds. For growth at lower temperatures, such a transition is delayed, as suggested by the observations of Fig. 2, where the RHEED patterns taken from films deposited at (a) $90 \mathrm{BLs}$, and (b) 630 and at temperatures of (i) $400^{\circ} \mathrm{C}$ and (ii) $650{ }^{\circ} \mathrm{C}$, respectively, are presented. It is seen that at $400^{\circ} \mathrm{C}$, at the thickness of $630 \mathrm{BLs}$ [Fig. 2(ib)], a polycrystalline film is evident (the ring patterns). At this thickness, on the other hand, the polycrystalline film grown at $650{ }^{\circ} \mathrm{C}$ has become single crystalline as suggested by the change of the ring-pattern [Fig. 2(iia)] to a spotty one [Fig. 2(iib)]. Obviously, high temperature is favored for better crystallinity of epitaxial $\mathrm{GaN}$ directly deposited on $\mathrm{Si}(111)$. Figure 2(ic) and 2(iic) show SEM micrographs of a $\sim 200 \mathrm{~nm}$ thick sample grown at 400 and $650{ }^{\circ} \mathrm{C}$, respectively, revealing the morphology of the two films. It can be observed that the crystalline grains size of the HT-film is 10 times larger than that of the LT-film.

We have then experimented the effect of Ga: $\mathrm{N}$ flux ratio on the surface and crystallinity of epitaxial GaN on $\operatorname{Si}(111)-(7 \times 7)$. It is found that despite the similar behavior of transformation of the initial diffusive diffraction pattern to a ring pattern with increasing deposition thickness, higher $\mathrm{Ga} / \mathrm{N}$ flux ratios will lead to delays of such transitions. In particular, growth under Ga-stable $(\mathrm{Ga} / \mathrm{N}>1)$ condition does not improve the morphology and crystallinity of epitaxial $\mathrm{GaN}$ on $\mathrm{Si}(111)$, contrasting the cases on other substrates, such as $\mathrm{SiC}$ and sapphire, for example. ${ }^{30-32}$ On the other hand, too low a flux ratio (e.g., $<0.7: 1)$ may cause partial nitridation of the substrate, forming amorphous $\mathrm{Si}_{x} \mathrm{~N}_{y}$ that may lead to facets and eventual degradation of crystal quality as evident from the SEM and XRD measurements. The optimal flux ratio is found to be Ga:N $\sim 0.77: 1$, which results in epilayers showing the narrowest FWHM in the XRD rocking curve measurements and better surfaces according to SEM examinations.

From the above RHEED observations, it seems that there is an initial amorphous layer growth stage upon GaN deposition on clean Si. Formation of such an amorphous-like layer may be related to the large lattice and thermal mismatch between $\mathrm{Si}$ and $\mathrm{GaN}$, and the amorphous-like layer, in which atoms are less regularly arranged and the lattices are more distorted than those of the crystalline substrate, may help to accommodate/relieve the strain in the system (i.e., there will be no coherency of the lattice at the interface). In fact, by the RHEED measurement of inplane lattice parameters of the epifilms immediately after the amorphous - crystalline transition, we note the films are almost strain-free, or at most, slightly tensile-strained. It seems that the majority of the strain is relived during the initial amorphous and/or polycrystalline layer growth stage.

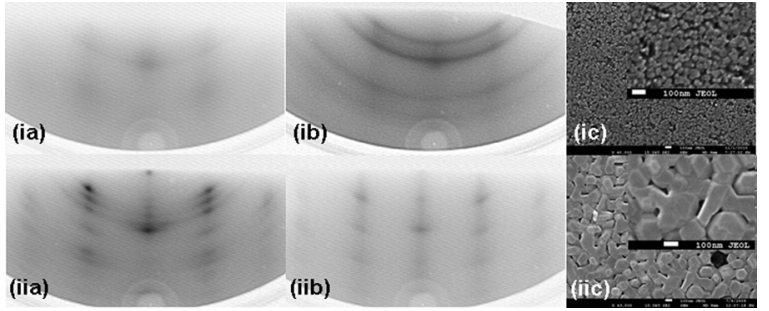

FIG. 2. RHEED patterns along GaN $[11 \overline{2} 0]$ of epitaxial GaN-on-Si(111) but at thicknesses of (a) 90 BLs and (b) 630 BLs. Panels (i) and (ii) are for films grown at 400 and $650^{\circ} \mathrm{C}$, respectively. (ic) and (iic) are SEM micrographs showing the surfaces of $\sim 200 \mathrm{~nm}$ thick GaN films grown on $\mathrm{Si}(111)$ at 400 and $650^{\circ} \mathrm{C}$, respectively. The insets show the same surfaces with higher magnification.

\section{B. Two-step growth: Effect of LT-buffer thickness}

As shown in Figs. 1 and 2 above, a high temperature leads to better crystallinity of epitaxial $\mathrm{GaN}$ on $\mathrm{Si}(111)$ for a given thickness of the films. However, both the RHEED and SEM experiments show the surfaces are rough and dominated by grains or islands, which is far from the ideal smooth morphology for application purposes. To improve the surfaces, we experiment a two-step growth procedure, where a LT-buffer layer is deposited prior to the HT film growth. The effect of the LT-buffer thickness is investigated and a minimal thickness of $\sim 3.3 \mathrm{~nm}$ is established for optimal epitaxy.

In Fig. 3, we present the RHEED patterns of epitaxial $\mathrm{GaN}$ at different thicknesses of deposition at $650^{\circ} \mathrm{C}$ but with a LT $\left(400^{\circ} \mathrm{C}\right)$ buffer of $\sim 6.6 \mathrm{~nm}$ thick. Clearly, the surface morphology improves with film thickness as suggested from the initially ring-like [Fig. 3(a)] to the intermediate spotty [Fig. 3(b)] and finally to a streaky pattern [Fig. 3(c)]. Note the appearance of the $(3 \times 3)$ [Fig. 3(c)] and $(6 \times 6)$ (not shown) patterns of the surfaces, which indicates the $\mathrm{N}$ polarity of the film. ${ }^{33}$ We have also measured the spacing $\boldsymbol{D}$ between the diffraction spots or streaks [refer to Fig. 3(d)] for lattice parameter and residual strain in film. The lateral width $\boldsymbol{W}$ of the (0004) spot or central diffraction streak is also measured for film's crystallinity and/or grain size. For

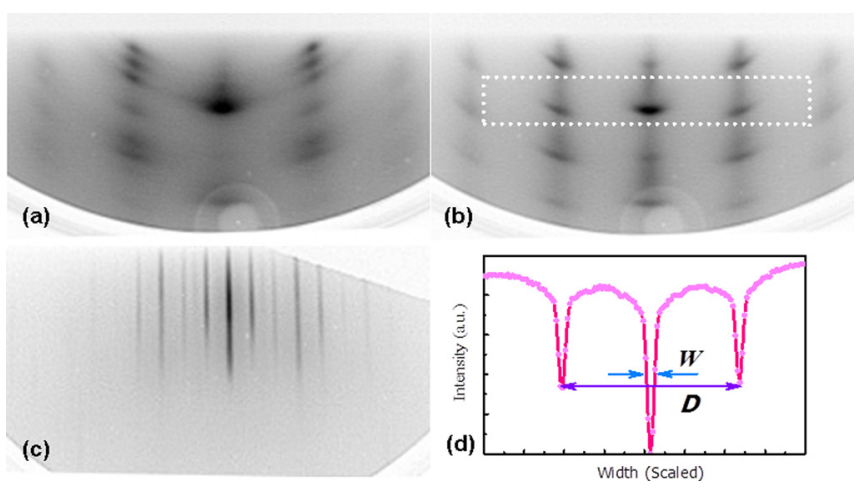

FIG. 3. (Color online) RHEED patterns along GaN [112̄0] of epitaxial GaN films deposited on $\mathrm{Si}(111)$ at $650^{\circ} \mathrm{C}$ (HT) but with a $\sim 6.6 \mathrm{~nm}$ thick $\mathrm{GaN}$ buffer deposited at $400^{\circ} \mathrm{C}$ (LT) underneath. The HT-film thickness for (a), (b), and (c) are 3, 36, and $250 \mathrm{~nm}$, respectively. Panel (d) shows the (average) intensity profile taken along lines in the region illustrated by the dashed box in (b), from which the values of $D$ and $W$ are measured. 
these, line profiles crossing the diffraction spots and/or streaks [dashed box in Fig. 3(b)] are first made, and the values of $D$ refer to the distance between $(01)$ and $(0 \overline{1})$ streaks while $\boldsymbol{W}$ 's are taken as the full width at half maximum (FWHM) of the intensity profile. Figure 4(a) summarizes the results for samples of different thicknesses, and the data of varying LT-buffer thickness are compared. For reference, samples grown directly on $\mathrm{Si}(111)$ at $650{ }^{\circ} \mathrm{C}$ without the LTbuffer are also shown (represented by open triangles in figure). The recorded evolutions of the lattice parameter $a \propto 1 / D$, where $a$ is the in-plane lattice constant of the epifilm, are also included in the figure, from which one observes that the majority of lattice misfit strain has been relieved in the very early stage of deposition and it remains approximately unchanged during later stage depositions for greater thicknesses. On the other hand, all the films appear to contain a minute amount of residual strain, although the amount of the residual strain is reduced in films with the LT-buffer. Second, when comparing the measured $W$ for samples with and without the LT-buffers, one observes apparent improvement of crystal quality by adopting the LT-buffer, as implied by the reduced $W$. Increasing the thickness of the LT-buffer $(d)$, the width of the diffraction spot/streak $(W)$ becomes narrower until $\boldsymbol{d}$ reaches $\sim 3.3 \mathrm{~nm}$, beyond which, further
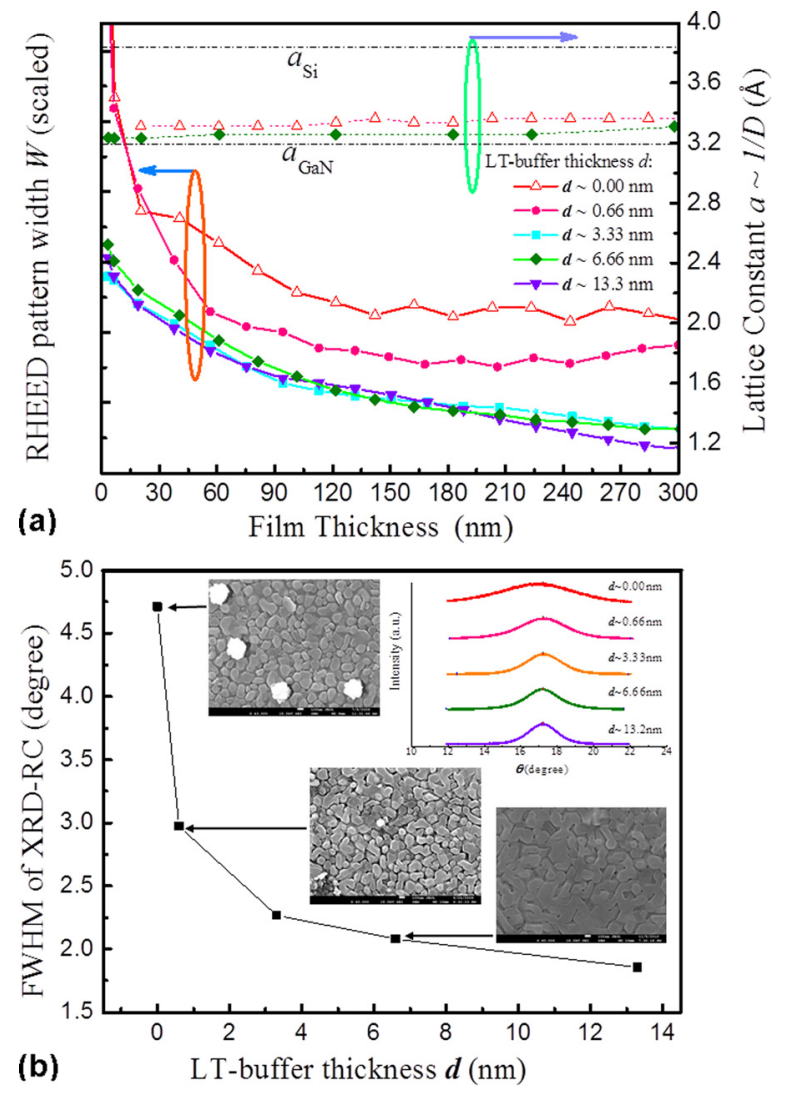

FIG. 4. (Color online) (a) Evolution of the RHEED pattern width $(W)$ as a function of HT-film thickness. Different curves show results for different LT-buffer thickness. The evolutions of in-plane lattice constant as derived from $D$ are also shown, comparing that of strain-free $\mathrm{Si}$ and $\mathrm{GaN}$ (dashdotted lines). (b) FWHM of the XRD rocking curve (RC) of $\mathrm{GaN}(0002)$ diffraction for $\sim 250 \mathrm{~nm}$ thick $\mathrm{GaN}$ films grown at $650^{\circ} \mathrm{C}$ but having different LT-buffer thicknesses. The inset shows the original XRD curves, and the SEM micrographs are for samples indicated by arrows. increasing $\boldsymbol{d}$ no longer brings in further obvious decrease of $W$. In all cases, the thicker the sample, the better the film's quality as judged by continuous narrowing of the diffraction patterns with increasing film thickness.

As is known, the value of $W$ of the RHEED reflects the crystallinity and/or grain sizes of the epifilm near the surface region. To determine the dominant factor affecting the changes in $W$, we carried out XRD rocking curve measurements of the epifilms having the same total thickness of $\sim 250 \mathrm{~nm}$ but using different thicknesses of the LT-buffers. Figure 4(b) summarizes the results, where the FWHM of $\mathrm{GaN}$ (0002) diffraction rocking curves (see inset) are plotted. SEM micrographs from the same set of samples are also shown in figure, depicting the grain sizes of the epifilms. As is evident, increasing the LT-buffer thickness, the FWHM of the XRD rocking curves becomes smaller, indicating continuous improvement of the crystallinity of the epifilms. Consistent with the RHEED data, significant effect occurs for the LT-buffers of $\leq 3.3 \mathrm{~nm}$ thick. Beyond $d \sim 3.3 \mathrm{~nm}$, the effect is not as dramatic. On the other hand, from the SEM images, one notes no significant improvement in lateral grain size by increasing $d$. Therefore, we assert that by incorporating a LT-buffer, crystal's structural quality is improved in terms of defect suppression rather than enlargement of grain sizes. This can be further supported by the TEM experiments of the samples as detailed below.

Recall that both the LT- and HT-depositions of GaN directly on $\mathrm{Si}(111)$ result in formation of amorphous layers according to the RHEED observations (i.e., diffusive patterns). This is further evidenced by the TEM measurements of the samples as presented in Fig. 5, where Figs. 5(a) and 5(b)

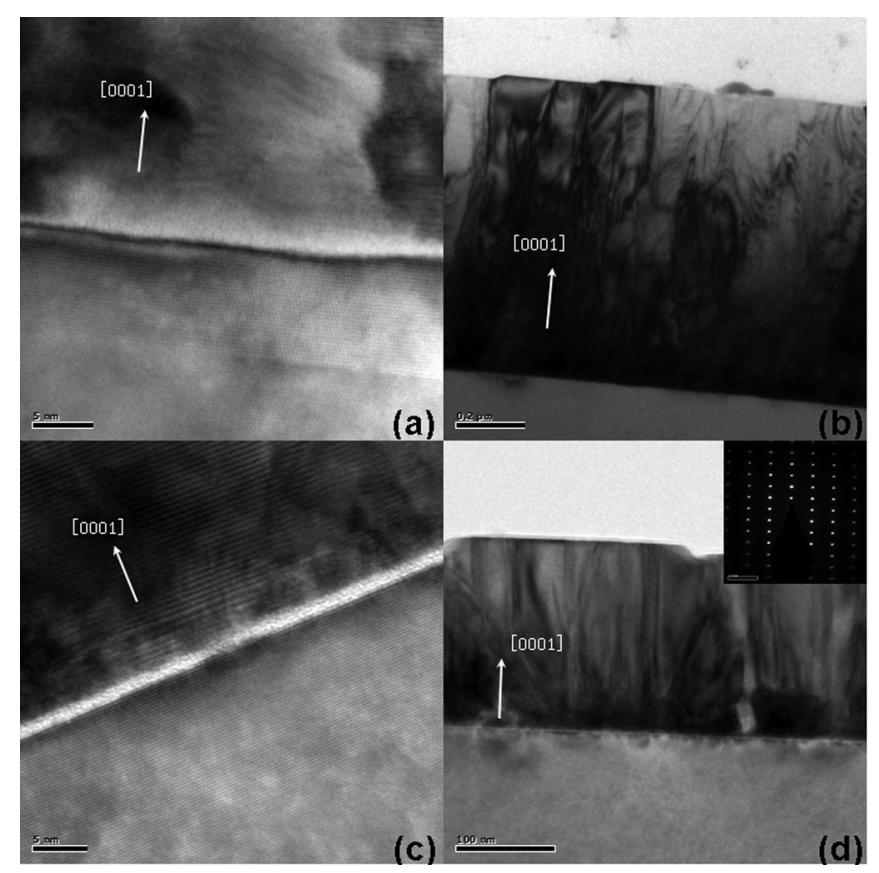

FIG. 5. TEM micrographs (zone axis: $\mathrm{Si}[110])$ of GaN-on-Si(111) samples grown at $650^{\circ} \mathrm{C}$ without $(\mathrm{a}, \mathrm{b})$ and with $(\mathrm{c}, \mathrm{d})$ a $\sim 6.6 \mathrm{~nm}$ thick LT-buffer. The high resolution images of (a) and (c) reveal the presence of amorphouslike interface layers, while in (b) and (d), threading defects in epifilms are revealed. The inset in (d) shows the selective area diffraction pattern of the sample. 
show cross-sectional TEM micrographs at different magnifications of a $\mathrm{GaN} / \mathrm{Si}$ sample grown at $650{ }^{\circ} \mathrm{C}$ without the LTbuffer, while Figs. 5(c) and 5(d) are the results of a sample having a $\sim 6.6$-nm-thick LT-buffer. First, from the highresolution TEM micrographs [Figs. 5(a) and 5(c)], one notes that for directly grown film without the LT-buffer, the bright amorphous-like layer appears thicker than the sample with the LT-buffer. Associated with this is a higher density of threading defects in the directly-grown film [Fig. 5(b)] and the larger residual strain [Fig. 4(a)]. So, adopting a LTbuffer seems to be advantageous to strain relaxation and improving crystal quality.

It will be interesting and important to examine in more detail of the identity of the bright amorphous-like layers at the heterointerface. First, we assert that they are indeed dominantly amorphous rather than a TEM artifact of a highly misoriented region. To ensure this, we carried out examinations of a specimen at varying rotation angles (up to $3^{\circ}$ ) relative to the e-beam of the TEM and found little change in structure and contrast of the bright amorphous-like region. We may thus rule out the effect of lattice rotation and/or distortion causing the amorphous-like feature in TEM. Particularly, we do not observe periodic lattice features in this region, being consistent with the assignment of an amorphous layer. This would agree with the diffusive RHEED patterns presented earlier. As for the composition of this region, due to its narrowness, an energy-dispersive $x$-ray spectroscopy measurement becomes unreliable. However, considering the highly reactive spices of $\mathrm{N}$ atoms and ions from the plasma source, formation of $\mathrm{Si}_{x} \mathrm{~N}_{y}$ is likely. On the other hand, as remarked earlier, we commenced the deposition immediately after the plasma was ignited, at which the $(7 \times 7)$ pattern of $\mathrm{Si}(111)$ remained visible in the RHEED, the effect of surface nitridation was minimized. We even attempted protecting the Si surface by depositing a coverage of $\mathrm{Ga}$ prior to $\mathrm{N}$ plasma ignition and observed little difference from that without the Ga predeposition. Added by the persistent diffusive RHEED pattern after a thickness of $\mathrm{GaN}$ deposition, we suggest the amorphous-like layer also contains a highly disordered or amorphous GaN.

The question is how a crystalline (though highly defected) film eventually form and grow on top of such an amorphous film. This is unclear at the moment, but we may make some speculations. Since the initial diffusive RHEED pattern lasted for a longer time period than the thickness of the TEM data would suggest, we assume there might be a solid state crystallization as the deposition proceeds, particularly at elevated temperatures. Such crystallization process may preferably happen at or near the surface region. Once crystalline GaN nuclei form, they will then act as the seeds for further growth of a crystalline film on top and catalyze further crystallization of the amorphous layer below. We tried to anneal the LT-buffer at $650{ }^{\circ} \mathrm{C}$ without deposition and observed a change of the RHEED from the initial diffusive pattern to rings and finally to spots, signaling a crystallization process by annealing itself. We may not even rule out the presence of small GaN crystallites in the amorphous layer itself, although our TEM studies have not provided a direct evidence for that. If such small crystallites exist, they can be the natural seeds for later stage growth of a crystalline film over the amorphous region.

\section{Transport properties of GaN/Si heterojunctions}

Measurements of the transport behavior across the heterojunctions of $\mathrm{GaN} / \mathrm{Si}$ have been made on samples grown using the two-step method. GaN films grown on both $p$ - and $n$-type $\operatorname{Si}(111)$ substrates are tested. The electrical carrier concentrations of the substrate as well as that of the GaN films are characterized by Hall effect measurements. We note that the thickness of the LT-buffer layer affects the background doping level. The thicker the LT-buffer, the lower the background doping is. Here, the $p$-type substrate has a hole concentration of $1.2 \times 10^{19} \mathrm{~cm}^{-3}$, and the $n$-type $\mathrm{Si}$ have electron concentrations of $2.3 \times 10^{19}\left(n^{+}\right)$and $\sim 2.0 \times 10^{15} \mathrm{~cm}^{-3}\left(n^{-}\right)$for the types (heavy and lightly doped) of wafers, respectively.

Figure 6(a) depicts typical $I-V$ characteristics of junctions of (i) $\mathrm{GaN} / p-\mathrm{Si}$, and (ii) $\mathrm{GaN} / n^{-}-\mathrm{Si}$, respectively. To our surprise, all the $\mathrm{GaN} / p$-Si junctions exhibit ohmic behavior, so are the $\mathrm{GaN} / n^{+}-\mathrm{Si}$ junctions (not shown). Only samples grown on lightly phosphor doped $\mathrm{Si}$, i.e., the $\mathrm{GaN} / n^{-}-\mathrm{Si}$ junction, exhibited the rectifying behavior. For the latter, it is also puzzling about the forward-bias direction, i.e., the $\mathrm{GaN}$ side is at the higher electrical potential. In some
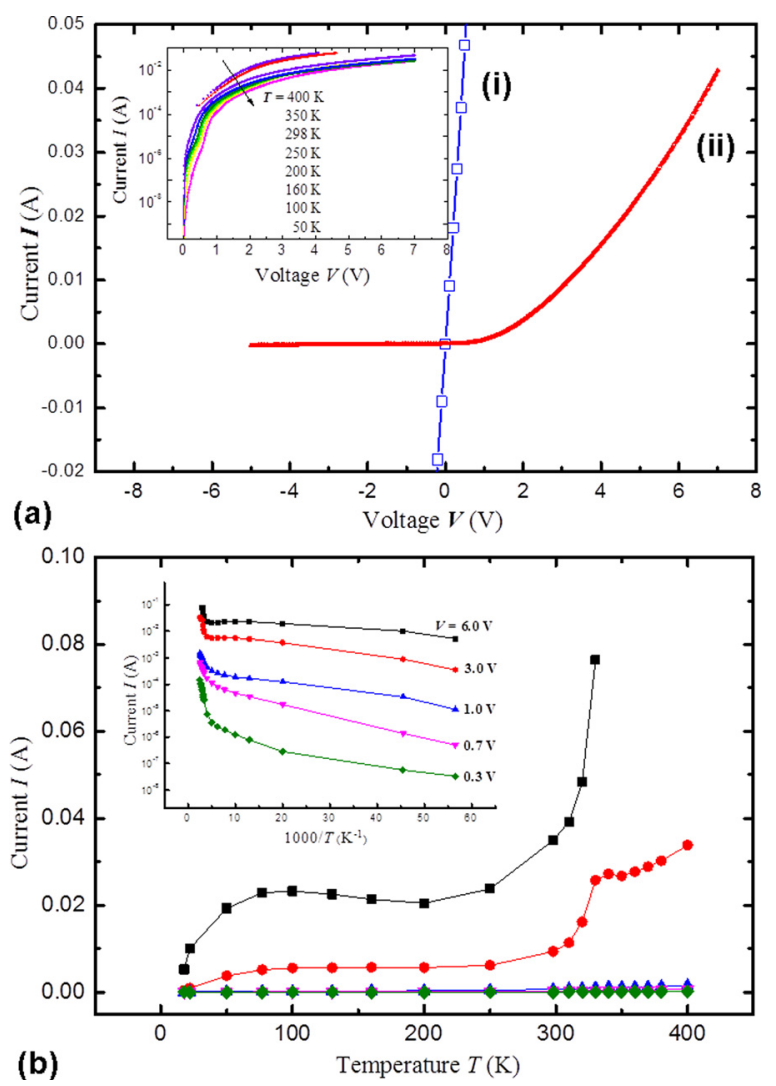

FIG. 6. (Color online) (a) Room-temperature $I-V$ characteristics of heterojunctions of (i) $\mathrm{GaN} / p$-Si (Al electrodes) and (ii) $\mathrm{GaN} / n^{-}-\mathrm{Si}$ (ITO and $\mathrm{Ag}$ electrodes for $\mathrm{GaN}$ and $n^{-}-\mathrm{Si}$, respectively). The inset shows, in a semilogarithm plot, the forward-bias $I-V$ data obtained at different temperatures between 18 and $400 \mathrm{~K}$. (b) Current-temperature $(I-T)$ dependence at different forward-bias conditions. The inset shows the same data in an Arrhenius plot. 
previous experiments of $\mathrm{GaN}$ growth on $\mathrm{Si}$ by metal organic vapor phase epitaxy at high temperatures $\left(\sim 1000^{\circ} \mathrm{C}\right)$, an effect of melt back etching of $\mathrm{Si}$, or some interdiffusion/ alloying of $\mathrm{Ga}$ and $\mathrm{Si}$ at the heterointerface was observed. ${ }^{34,35}$ For the MBE films grown at low temperatures, it is unclear if a similar effect takes place, though in the TEM micrographs we do observe some contrast difference on the $\mathrm{Si}$ side between regions near the interface and in bulk. If $\mathrm{Ga}$ diffusion in $\mathrm{Si}$ does occur, it will generate $p$-type doping in the otherwise $n^{-}$-type substrate, resulting in a $p-n$ junction. However, even if it is the case, the obtained $I-V$ data remain difficult to understand, not to say Ga diffusion is very limited at the temperatures of MBE process $\left(650^{\circ} \mathrm{C}\right)$ according to the literature. ${ }^{36-38}$

In the inset of Fig. 6(a), we show the $I-V$ data measured at different temperatures $(18-400 \mathrm{~K})$ and plotted in a semilogarithm plot. As is evident, the forward-bias current is far from the simple exponential relation expected from simple thermionic emission or tunneling mechanisms, ${ }^{39-42}$ even if the series resistance effect is taken into account. Despite this, we attempted to fit the data in the low-current condition by a relation $I=I_{0} \exp (q V / \eta k T)$, where $I_{0}$ and $\eta$ are the saturation current and ideality factor, respectively, and $q, k$, and $T$ stand for the magnitude of electron charge, the Boltzmann constant and absolute temperature, respectively. The fitting parameter of $\eta$ was found to be in the range of a few tens, signifying the non-conformity of the transport behavior to the above mentioned mechanisms.

To provide further details of the $I-V$ characteristics, we present in Fig. 6(b) a temperature dependence of the forward-bias current at different bias voltage conditions, while the inset shows the same data in an Arrhenius plot. It can be seen that two main temperature regimes may be distinguished. At low temperatures $(<240 \mathrm{~K})$, the current is only weakly dependent on temperature. The $\log (I) \sim 1 / T$ relation may be approximated by a straight line, and the slope does not seem to depend on the bias. At high temperatures, on the other hand, a much stronger dependence between $I$ and $T$ is noted. So, we may infer that completely different mechanisms dominate the transport behavior in the two temperature regimes. Due to the presence of a thin amorphous layer at the heterointerface of $\mathrm{GaN} / \mathrm{Si}$ and high densities of defects such as dislocations, the exact mechanism(s) require more detailed analyses and further experimental investigations.

\section{SUMMARY}

A systematic investigation of GaN epitaxial growth on $\mathrm{Si}(111)$ without a priori surface treatment has been carried out. N-stable flux condition and relative high growth temperature favor better films. However, a two-step procedure, where a LT-buffer precedes HT-film deposition, seems essential to achieving a smooth surface as well as better crystallinity of an epitaxial GaN. Irrespective of the growth conditions and the strategy (direct versus two-step growth), an amorphous interface layer forms. Transport measurements of the heterojunctions reveal ohmic behavior for both $\mathrm{GaN} / p-\mathrm{GaN}$ and $\mathrm{GaN} / n^{+}-\mathrm{Si}$ junctions, but rectifying characteristics for $\mathrm{GaN} / n^{-}$-Si junctions. Temperature dependence of the $I-V$ data reveals complications of the transport properties, which cannot be explained by simple processes.

\section{ACKNOWLEDGMENTS}

We acknowledge the help from W. K. Ho in the growth and SEM experiment, A. M. C. Ng for electrode making and preliminary transport measurements, and Y.F. Chan for providing the TEM data. This work was financially supported by a grant from the Research Grant Council of the Hong Kong Special Administrative Region, China, under the grant No. 7048/08P.

${ }^{1}$ S. Nakamura, M. Senoh, S. Nagahama, N. Iwasa, T. Yamada, T. Matsushita, Y. Sugimoto, and H. Kiyoku, Appl. Phys. Lett. 70, 1417 (1997).

${ }^{2}$ J. I. Pankove and T. D. Moustakas, Gallium Nitride (GaN) I (Academic, San Diego, 1998).

${ }^{3}$ J. I. Pankove and T. D. Moustakas, Gallium Nitride (GaN) II (Academic, San Diego, 1999).

${ }^{4}$ S. Nakamura and G. Fasol, The Blue Laser Diode : GaN Based Light Emitters and Lasers (Springer, Berlin, 1997).

${ }^{5}$ T. Yamaguchi, C. Morioka, K. Mizuo, M. Hori, T. Araki, Y. Nanishi, and A. Suzuki, 2003 International Symposium on Compound Semiconductors: Post-Conference Proceedings, p. 214 (2004).

${ }^{6}$ J. Wu, W. Walukiewicz, K. Yu, W. Shan, J. Ager III, E. Haller, H. Lu, W. Schaff, W. Metzger, and S. Kurtz, J. Appl. Phys. 94, 6477 (2003).

${ }^{7}$ H. Hamzaoui, A. S. Bouazzi, and B. Rezig, Sol. Energy Mater. Sol. Cells 87, 595 (2005).

${ }^{8}$ S. W. Feng, C. M. Lai, C. H. Chen, W. C. Sun, and L. W. Tu, J. Appl. Phys. 108, 093118 (2010).

${ }^{9}$ M. Yamaguchi, K. Nishimura, T. Sasaki, H. Suzuki, K. Arafune, N. Kojima, Y. Ohsita, Y. Okada, A. Yamamoto, T. Takamoto, and K. Araki, Sol. Energy 82, 173 (2008).

${ }^{10}$ X. Chen, K. D. Matthews, D. Hao, W. J. Schaff, and L. F. Eastman, Phys. Status Solidi A 205, 1103 (2008).

${ }^{11}$ O. Jani, C. Honsberg, Y. Huang, J. O. Song, I. Ferguson, G. Namkoong, E. Trybus, A. Doolittle, and S. Kurtz, Conference Record of the 2006 IEEE 4th World Conference on Photovoltaic Energy Conversion (IEEE, New York, 2006), Vols. 1 and 2, p. 20.

${ }^{12}$ C. H. Henry, J. Appl. Phys. 51, 4494 (1980).

${ }^{13}$ A. Yamamoto, M. Tsujino, M. Ohkubo, and A. Hashimoto, Sol. Energy Mater. Sol. Cells 35, 53 (1994).

${ }^{14}$ E. Trybus, G. Namkoong, W. Henderson, S. Burnham, W. A. Doolittle, M. Cheung, and A. Cartwright, J. Cryst. Growth 288, 218 (2006).

${ }^{15}$ K. Mizuo, T. Yamaguchi, Y. Saito, T. Araki, and Y. Nanishi, GaN and Related Alloys -2002, Materials Research Society Proceeding, 743, 719 (2003).

${ }^{16}$ I. Mahboob, T. D. Veal, C. F. McConville, H. Lu, and W. J. Schaff, Phys. Rev. Lett. 92, 036804 (2004).

${ }^{17}$ K. A. Rickert, A. B. Ellis, F. J. Himpsel, H. Lu, W. Schaff, J. M. Redwing, F. Dwikusuma, and T. F. Kuech, Appl. Phys. Lett. 82, 3254 (2003).

${ }^{18}$ D. Kapolnek, S. Keller, R. Vetury, R. D. Underwood, P. Kozodoy, S. P. D. Baars, and U. K. Mishra, Appl. Phys. Lett. 71, 1204 (1997).

${ }^{19}$ O. H. Nam, M. D. Bremser, T. S. Zheleva, and R. F. Davis, Appl. Phys. Lett. 71, 2638 (1997).

${ }^{20}$ R. F. Davis, T. Gehrke, K. J. Linthicum, P. Rajagopal, A. M. Roskowski, T. Zheleva, E. A. Preble, C. A. Zorman, M. Mehregany, U. Schwarz, J. Schuck, and R. Grober, MRS Internet J. Nitride Semicond. Res. 6, 1 (2001).

${ }^{21}$ Y. E. Romanyuk, D. Kreier, Y. Cui, K. M. Yu, J. W. Ager, and S. R. Leone, Thin Solid Films 517, 6512 (2009).

${ }^{22}$ T. Yodo, H. Ando, H. Tsuchiya, D. Nosei, M. Shimeno, and Y. Harada, J. Cryst. Growth 227, 431 (2001).

${ }^{23}$ H. X. Liu, Z. Z. Ye, H. X. Zhang, and B. H. Zhao, Mater. Res. Bull. 35, 1837 (2000)

${ }^{24}$ C. L. Wu, J. C. Wang, M. H. Chan, T. T. Chen, and S. Gwo, Appl. Phys. Lett. 83, 4530 (2003).

${ }^{25}$ F. R. Hu, K. Ochi, Y. Zhao, B. S. Choi, and K. Hane, J. Cryst. Growth 294, 197 (2006). 
${ }^{26}$ E. Feltin, B. Beaumont, M. Laugt, P. de Mierry, P. Vennegues, H. Lahreche, M. Leroux, and P. Gibart, Appl. Phys. Lett. 79, 3230 (2001).

${ }^{27}$ A. Able, W. Wegscheider, K. Engl, and J. Zweck, J. Cryst. Growth 276, 415 (2005).

${ }^{28}$ S. M. Seutter, M. H. Xie, W. K. Zhu, L. X. Zheng, H. S. Wu, and S. Y. Tong, Surf. Sci. 445, L71 (2000).

${ }^{29}$ H. Ahn, C. L. Wu, S. Gwo, C. M. Wei, and Y. C. Chou, Phys. Rev. Lett. 86, 2818 (2001).

${ }^{30}$ C. D. Lee, V. Ramachandran, A. Sagar, R. M. Feenstra, D. W. Greve, W. L. Sarney, L. Salamanca-Riba, D. C. Look, S. Bai, W. J. Choyke, and R. P. Devaty, J. Electron. Mater. 30, 162 (2001).

${ }^{31}$ J. M. Myoung, O. Gluschenkov, K. Kim, and S. Kim, J. Vac. Sci. Technol. A 17, 3019 (1999).

${ }^{32}$ E. C. Piquette, P. M. Bridger, R. A. Beach, and T. C. McGill, MRS Internet J. Nitride Semicond. Res. 4, G3.77 (1999).

${ }^{33}$ A. R. Smith, R. M. Feenstra, D. W. Greve, M. S. Shin, M. Skowronski, J. Neugebauer, and J. E. Northrup, Appl. Phys. Lett. 72, 2114 (1998).

${ }^{34}$ H. Ishikawa, K. Yamamoto, T. Egawa, T. Soga, T. Jimbo, and M. Umeno, J. Cryst. Growth 190, 178 (1998).
${ }^{35}$ K. Takemoto, H. Murakawi, T. Iwamoto, Y. Matsuo, Y. Kangawa, Y. Kumagai, and A. Koukitu, Jpn. J. Appl. Phys., Part 2 45, L478 (2006).

${ }^{36}$ V. Fiorentini, C. Melis, and G. M. Lopez, Appl. Phys. Lett. 85, 4902 (2004).

${ }^{37}$ E. Calleja, M. A. Sanchez-Garcia, D. Basak, F. J. Sanchez, F. Calle, P. Youinou, E. Munoz, J. J. Serrano, J. M. Blanco, C. Villar, T. Laine, J. Oila, K. Saarinen, P. Hautojarvi, C. H. Molloy, D. J. Somerford, and I. Harrison, Phys. Rev. B 58, 1550 (1998).

${ }^{38}$ S. Haridoss, F. Beniere, M. Gauneau, and A. Rupert, J. Appl. Phys. 51, 5833 (1980).

${ }^{39}$ Z. Hassan, S. S. Ng, G. Chew, F. Yam, M. Abdullah, M. Hashim, K. Ibrahim, and M. Kordesch, in Growth and Properties of $\mathrm{GaN} / \mathrm{Si}$ Heterojunction (Pergamon-Elsevier Science Ltd, Oxford, 2005), p. 531 .

${ }^{40}$ Z. Hassan, Y. C. Lee, F. K. Yam, K. Ibrahim, M. E. Kordesch, W. Halverson, and P. C. Colter, Solid State Commun. 133, 283 (2005).

${ }^{41}$ Ş. Aydoğan, M. Sağlam, and A. Türüt, J. Phys.: Condens. Matter 18, 2665 (2006).

${ }^{42}$ S. M. Sze, Physics of Semiconductor Devices, 2nd ed. (Wiley, New York, 1981). 